\title{
Entre expertises et jeux d'acteurs : la trame verte et bleue du Grenelle de l'environnement
}

\author{
Ruppert Vimal ${ }^{1}$, Raphaël Mathevet ${ }^{2}$, Laura Michel ${ }^{3}$ \\ 1 Écologue, CNRS, UMR5175 Centre d'écologie fonctionnelle et évolutive, 34293 Montpellier cedex 5, France \\ 2 Géographe, CNRS, UMR5175 Centre d'écologie fonctionnelle et évolutive, 34293 Montpellier cedex 5, France \\ 3 Politiste, UM1-CNRS, UMR5112 Centre d'étude politique d'Europe latine, 34060 Montpellier cedex 1, France
}

En partant de l'observation de l'une des scènes du Grenelle de l'environnement, les auteurs posent une vieille question, familière à la revue, qui est celle de la place de la science, et plus précisément de l'expertise, dans un dispositif de concertation publique. Mais leur analyse renouvelle cette problématique en interrogeant la combinatoire effective entre des connaissances issues de l'académie et celles issues de l'expertise collective. Les asymétries de compétences et les dispositions des acteurs à coopérer apparaissent alors tributaires de la capacité d'un collectif participatif à bien distinguer ce qu'il peut attendre, d'une part, de l'expertise académique, d'autre part, d'une expertise collective ouvrant sur une démarche plus pragmatique.

La Rédaction

\section{Mots-clés :}

Grenelle

de l'environnement ; réseau écologique ; trame verte et bleue ; dispositif participatif expertise scientifique

\begin{abstract}
Résumé - Dans cet article, nous analysons le processus délibératif chargé de définir une politique publique de trame verte et bleue (TVB) en tant que stratégie de conservation intégrée, dans le cadre du Grenelle de l'environnement. Nous montrons que l'expertise scientifique est un enjeu central dans le processus social aboutissant à une représentation de la TVB et à un cadre d'action publique. Le forum hybride en charge de son élaboration n'était pas isolé et la définition de la TVB et du cadre d'action publique qui en résulte est largement dépendante d'interactions, de jeux d'acteurs, de contraintes historiques ou encore d'un cadre législatif supranational qui dépassent largement l'arène participative et questionnent son autonomie. Nous soulignons ici l'enjeu de la place accordée à l'expertise académique pluridisciplinaire pour donner du sens au collectif et ainsi lui permettre de faire «cause commune ».
\end{abstract}

\section{Keywords:}

Grenelle Environment

Forum; ecological network; Green and Blue Infrastructure; participatory device; scientific expertise

\begin{abstract}
Between scientific expertise and power games: the Green and Blue Infrastructure of the Grenelle Environment Forum. In this paper we investigate the deliberative process charged with defining an integrated conservation policy within the framework of the French Grenelle Environment Forum, namely the Green and Blue Infrastructure ("trame verte et bleue" [TVB]). We point out the central importance of scientific expertise in the social process leading to the representation of TVB and the definition of a framework for public action. The hybrid forum in charge of its characterization did not function in isolation. The TVB definition and the resulting public action framework are dependent on social interactions and networks, historical constraints or supranational legal frameworks, which transcend the participatory arena and question its autonomy. We emphasize the importance of the place given to interdisciplinary academic expertise as determinant in creating sense for the group and orienting it toward a "common cause".
\end{abstract}

\section{Introduction}

Les rapports qu'entretiennent la science et la politique ont très tôt interrogé les sociologues (Weber, 1959). Si les outrages de la science au débat démocratique sont souvent dénoncés (Habermas, 1973 ; Touraine, 1980), l'analyse des politiques publiques s'intéresse aussi au transfert de certains concepts des champs scientifiques

Auteur correspondant : R. Mathevet, raphael.mathevet@cefe.cnrs.fr 
vers les champs sociaux et politiques et aux simplifications dont ils sont l'objet pour devenir de simples doctrines ou images faisant sens, fort éloignées des concepts scientifiques d'origine (Muller, 1985 ; Jobert, 1994). De manière générale, les approches cognitivistes prennent leur distance avec une vision rationaliste ou « problem-solving » de l'action publique pour leur préférer une approche constructiviste. Muller (1995) définit une politique publique comme un lieu où une société construit son rapport au monde. Comprendre une politique publique, c'est alors s'intéresser aux processus sociaux qui façonnent les représentations dominantes d'une société à un moment donné, désignant les problèmes légitimes à traiter et la manière d'agir à leur encontre.

Mais si la politique publique est ainsi un moyen de produire du sens, elle est aussi un instrument de pouvoir. Il importe de souligner que les idées ne sont pas désincarnées : elles sont portées par des acteurs sociaux aux intérêts conflictuels qui luttent pour imposer la légitimité de leurs idées, mais aussi celles du groupe qu'ils représentent. Ainsi une politique publique fabrique des images, mais distribue aussi les fonctions et les pouvoirs entre acteurs concernés au travers d'un processus d'ajustement et d'articulation entre des intérêts et buts individuels différents et avec d'autres plus collectifs (Friedberg, 1993). Analyser les politiques publiques nécessite donc de s'intéresser aux dynamiques collectives au sein desquelles expertises et jeux de pouvoir sont socialement situés et s'entremêlent.

En s'inscrivant contre l'idée dominante d'une science coupée de la société ou d'une connaissance soustraite à un modèle unique d'activité scientifique, la sociologie de l'innovation offre un cadre réflexif pour analyser les rapports entre science, politique et société. Elle souligne par ailleurs que, si l'innovation s'impose, ce n'est pas en raison de sa qualité mais du fait du processus qui a supporté cette innovation (Callon et Latour, 1991).

Dans un monde où l'incertitude ne trouve plus de réponse dans le religieux ou les grands récits idéologiques, où les connaissances se révèlent partielles et incertaines, et les enjeux de plus en plus complexes, les rapports $\mathrm{qu}^{\prime}$ entretiennent science et politique ne peuvent plus se maintenir, le volontarisme politique comme l'activité scientifique ne peuvent plus être les seuls fondements légitimes de l'action publique (Callon et al., 2001).

Il s'agit dès lors de définir un cadre interprétatif des connaissances disponibles qui rende visibles les incertitudes et qui permette simultanément de confronter les valeurs et représentations des acteurs pour faire émerger de nouvelles perspectives (Roqueplo, 1997). Ainsi les concepts de science post-normale, de science contextualisée ou « de mode $2^{1}$ » (Funtowicz et Ravetz, 1993 ; Nowotny et al., 2001 ; Barré, 2004 ; Van der Windt et Swart, 2008), supposent que l'élaboration de la décision conduit les acteurs du forum hybride (Callon, 1988) à produire aussi de nouvelles connaissances, pragmatiques et fondées pour l'action. En confrontant au territoire les connaissances « de mode 1 », issues de la science normale, elles les dépassent en intégrant en outre le résultat des interactions sociales inhérentes au processus qui mène à la décision. Ce qui signifie que la qualité de la connaissance et sa robustesse sociale sont plus que jamais relatives à la qualité du dialogue (Habermas, 1987 ; Stengers, 2002). En reconnaissant la pluralité des légitimités d'action, le dispositif sociotechnique mis en place doit en effet permettre d'élaborer de façon pragmatique une vision partagée de l'action publique à travers un processus d'apprentissage collectif (ComMod, 2009 ; Habermas, 1987). Sur la base de ce postulat, cet article ${ }^{2}$ analyse la construction d'une politique publique de conservation intégrée de la biodiversité en France - la « trame verte et bleue » (TVB) - qui fait suite à un engagement du Grenelle de l'environnement. Nous nous interrogeons sur la place et la nature de l'expertise dans cette démarche originale et voulue participative: Qui représente l'expertise ? Comment les différents acteurs utilisent-ils l'expertise pour défendre leurs intérêts? Le répertoire d'expertise est-il accessible à tous les groupes d'intérêts de la même façon ? Comment cela influence-til en retour la qualité du dialogue et la nature du cadre $\mathrm{d}$ 'action publique qui en émergent?

\section{Le Grenelle de l'environnement et le comité opérationnel TVB}

En 2007, le gouvernement français a lancé une démarche originale intitulée "Grenelle de l'environnement» qui, autour d'un processus de négociation associant cinq collèges (collectivités, État, syndicats, entreprises et associations), avait pour objectif d'élaborer une nouvelle stratégie et un plan d'action national en

\footnotetext{
1 La science dite « de mode 2 » est dictée par le contexte, centrée sur le problème à résoudre et interdisciplinaire, tandis que la science « de mode 1 » est plutôt entreprise par le chercheur et la production de connaissance, basée sur une ou quelques disciplines (Nowotny et al., 2001). Ainsi, avec le mode 2, le contexte d'application des connaissances (inter- ou transdisciplinaire) écarte les seuls intérêts académiques (disciplinaires), l'organisation et la production de la connaissance deviennent sensiblement moins hiérarchiques et institutionnalisées. Enfin, la responsabilité sociale de la science prend une place importante aux dépens de son autonomie traditionnelle.

2 Cette étude a été financée par l'Agence nationale de la recherche (contrat $\mathrm{n}^{\circ} 05-\mathrm{BDIV}-014, \mathrm{ABIME)}$, l'Union européenne (contrat 226852, SCALES) et la région LanguedocRoussillon.
} 
matière environnementale. Dans ce cadre, le groupe chargé de la thématique biodiversité a proposé de « construire la Trame verte nationale, tissu vivant du territoire, qui assure les continuités et les proximités entre milieux naturels permettant aux espèces de circuler et d'interagir et aux écosystèmes de fonctionner » (MEDAD, 2007, p. 5). À l'issue des tables rondes, des comités opérationnels (COMOP) ont vu le jour ${ }^{3}$ afin $\mathrm{d}^{\prime}$ assurer une mise en œuvre opérationnelle des conclusions et relevés de décision du Grenelle. Le COMOP n ${ }^{\circ} 11$, en charge de la TVB, a dès lors bénéficié d'un mandat exceptionnellement long de deux ans, justifié par le caractère « complexe et innovant » de la TVB.

Sous la tutelle d'un sénateur socialiste de la région Nord-Pas-de-Calais, deux personnes ont assuré la direction et l'animation du COMOP TVB : le délégué au développement durable et à l'expertise du Muséum national d'histoire naturelle (MNHN) et le sous-directeur des espaces naturels au ministère de l'Écologie, de l'Énergie, du Développement durable et de la Mer (MEEDDM). Le COMOP a réuni une quarantaine de personnes appartenant à diverses structures: France Nature Environnement (FNE), l'Assemblée permanente des chambres d'agriculture (APCA), la Fédération nationale des syndicats d'exploitants agricoles (FNSEA), la Fédération des conservatoires d'espaces naturels (FCEN), la Fédération des parcs naturels régionaux de France (FPNRF), les Forestiers privés de France (FPF), la Fédération nationale pour la pêche en France, la Fédération nationale des chasseurs, le Mouvement des entreprises de France (MEDEF), l'Association des communes urbaines de France, l'Office national des forêts (ONF), l'Assemblée des communautés de France, Réserves naturelles de France, la Fondation Nicolas Hulot (FNH), ainsi qu'un grand nombre de fonctionnaires des services du MEEDDM ou d'autres ministères (Agriculture et Transports, notamment). Les représentants des départements et des régions, bien qu'invités, n'ont jamais participé au dispositif. Dès la première réunion, l'ensemble des participants soulignait que cette absence était problématique dans la mesure où les collectivités territoriales seraient des acteurs-clés de la mise en œuvre de la TVB. Notons que l'équipe TVB du ministère s'est étoffée et mise en place tout au long de la procédure. Une personne a été désignée pour être en charge dela politique TVB à long terme et deux autres agents ont appuyé le pilotage duCOMOP. Un groupe d'experts scientifiques s'est rapidement constitué autour de cette équipe, associant l'Office national de l'eau et des milieux aquatiques (ONEMA), le Service d'études sur les transports, les

\footnotetext{
3 Le processus «Grenelle de l'environnement » est donc constitué de deux phases : les tables rondes qui ont eu lieu à la fin de l'année 2007 et les comités opérationnels mis en place l'année suivante. Notre analyse porte spécifiquement sur un des comités opérationnels. Pour une analyse de la première phase du Grenelle, voir Lascoumes (2011).
}

routes et leurs aménagements (SETRA), le MNHN et piloté par le Cemagref ${ }^{4}$, institut de recherche en sciences et technologies pour l'environnement et l'agriculture.

Durant deux ans, le COMOP s'est réuni en plénière en moyenne une fois tous les quarante-cinq jours, ainsi que lors de nombreuses séances des groupes de travail. La mission du COMOP a consisté à définir les objectifs globaux de la TVB, à clarifier ce qui la constitue et les éléments de sa représentation, puis à identifier et proposer des outils pour la mettre en œuvre. Ces deux années de travail se concrétisent ainsi autour de divers documents :

- la partie TVB des textes de loi Grenelle 1 et 2 ;

- des supports de communication qui seront utilisés pour présenter la TVB aux différents publics concernés ;

- des rapports d'expertises, notamment sur les outils contractuels à destination de la TVB, l'intégration de la TVB dans les documents d'urbanisme, les enjeux socioéconomiques relatifs à la TVB ;

- un guide méthodologique intitulé Orientations nationales pour la préservation et la remise en bon état des continuités écologiques 5 .

Ce guide, qui sera traduit en décrets par la suite, est la principale production du COMOP et se décline en trois parties. Le premier volet (guide 1), à destination des élus, présente principalement les grandes orientations nationales et les choix stratégiques pour la TVB (AllagDhuisme et al., 2010a). Le second volet (guide 2) est un appui à la mise en œuvre de la TVB et à l'élaboration des schémas régionaux de cohérence écologique ; il s'adresse principalement aux responsables des services ayant en charge la mise en œuvre de la politique TVB (AllagDhuisme et al., 2010b). Enfin, le troisième volet (guide 3) traite de l'impact des infrastructures linéaires et s'adresse aux services de l'État et aux autres établissements publics qui en ont la charge (Allag-Dhuisme et al., 2010c).

Notre analyse se fonde sur la participation du premier auteur aux réunions plénières du COMOP (une quinzaine), ainsi que sur les comptes rendus et productions des différents groupes de travail et des entretiens semidirectifs avec les principaux protagonistes.

\section{La TVB ou la construction d'une nouvelle représentation de la protection de la nature}

Affichant le besoin d'une " préservation globale de la biodiversité et non plus seulement de ses éléments les

\footnotetext{
4 NB : le Cemagref (Centre national du machinisme agricole, du génie rural et des eaux et forêts) est devenu depuis 2011 l'IRSTEA (Institut national de recherche en sciences et technologies pour l'environnement et l'agriculture).

5 Cf. http://www.trameverteetbleue.fr/documentation-outils/ outils-methodes/productions-comite-operationnel-trame-vertebleue-issu-grenelle
} 
plus remarquables et emblématiques ou les plus menacés » (Allag-Dhuisme et al., 2010a, p. 5), la TVB semble s'inscrire en rupture avec les politiques de conservation ayant prévalu jusqu'à présent. Dans un but pédagogique, le guide mentionne (ibid.) : «Il faut donc commencer par renforcer le tissu vivant du territoire en rétablissant, à l'image du système sanguin pour le corps humain, des flux entre des organes vitaux - les zones de plus haute valeur écologique - de façon à ce que vive tout l'organisme - c'est-à-dire le tissu vivant du territoire. C'est le but de la Trame verte et bleue nationale. » Tout au long du guide, l'objet TVB reste cependant équivoque : il est tantôt perçu comme un état de fait, une réalité à protéger, tantôt défini comme un outil d'aménagement du territoire. Il s'agit de diagnostiquer la TVB ou de l'élaborer ; la TVB « doit constituer l'infrastructure naturelle du territoire sur laquelle doit s'inventer un aménagement durable à partir d'une organisation intelligente et économe de l'espace " (ibid.). Il semble par ailleurs que la définition et la représentation cognitive de la TVB soient clairement liées à la question de son identification spatiale, qui apparaît comme la condition sine qua non de sa mise en œuvre. Ainsi, le guide propose qu'elle soit " constituée de trois [...] éléments principaux baptisés, par souci de simplicité : les réservoirs de biodiversité et les corridors écologiques s'appliquant plus particulièrement aux milieux terrestres mais aussi humides, et enfin les cours d'eau » (Allag-Dhuisme et al., 2010b, p. 6). Cette représentation est ensuite justifiée (ibid., p. 7) : «La gestion des deux composantes de la Trame verte et bleue doit permettre aux espèces d'assumer leur fonction : nutrition, reproduction et repos pour les réservoirs de biodiversité, dispersion et migration pour les corridors. »

Dans un travail antérieur (Vimal et al., 2012), nous avons exposé comment la transmission d'un modèle scientifique (le modèle « taches-corridors-matrice ») de la communauté scientifique (à laquelle il permet de tester des hypothèses) à la société (où il devient l'unique représentation d'un objet particulièrement complexe) suppose de considérer que le maintien d'un maillage écologique passe principalement par les capacités de déplacement des espèces d'un patch à un autre à l'échelle du paysage et relève ainsi $\mathrm{d}^{\prime}$ une simplification conséquente de concepts écologiques complexes (Boitani et al., 2007).

Traduction non contestée des connaissances scientifiques, le modèle est, dans le cadre du COMOP, le moyen de contourner la complexité de l'objet en question et les controverses qui l'entourent au nom d'un besoin d'opérationnalité. Bien qu'inscrit dans la loi (loi n ${ }^{\circ} 2010-788$ du 12 juillet 2010), il reste une "boîte noire " (Callon et al., 2001) qui devient le support induit de toutes les préconisations d'identification et de gestion de la TVB. Le volet 2 du guide (Allag-Dhuisme et al., 2010b) propose toute une gamme de techniques issues de divers projets aux échelles variées pour identifier les différents constituants de la TVB. Tout en laissant le choix de la méthode au niveau régional, où régions et services de l'État doivent coélaborer un schéma régional de cohérence écologique, le guide mentionne des critères qui doivent obligatoirement être intégrés en amont ou en aval pour s'assurer de la cohérence du réseau écologique national résultant.

Le COMOP ancre de fait la TVB dans une démarche technique au sein de laquelle la représentation cartographique devient le pilier de la nouvelle stratégie. La cartographie des connectivités écologiques renvoie évidemment à la question de la définition des processus écologiques, focalisée ici sur leur dimension spatiale. Cette traduction spatiale de la TVB, censée permettre $\mathrm{d}$ 'identifier où débute et où s'arrête le réseau, permet in fine de se centrer sur l'enjeu de la définition des limites des zonages susceptibles de devenir totalement ou en partie opposables dans les documents d'urbanisme.

\section{Du rôle de l'expertise académique}

Face à la complexité inhérente au sujet traité et au caractère peu populaire et souvent contesté des politiques environnementales, l'enjeu a été pour le COMOP de produire un cadre d'action publique qui, comme l'a précisé le sénateur dès le début des travaux, soit « un compromis entre acceptabilité et science ${ }^{6} »$. La connaissance scientifique, dans le cadre de sa mobilisation pour l'action, est donc largement confrontée, d'une part, à sa contingence et à sa complexité et, d'autre part, aux contraintes sociales du territoire. Nous constatons que l'expertise scientifique s'est exprimée sous une grande diversité de formes au cours des deux années de mandat du COMOP, ce qui a rendu les processus conduisant au compromis entre expertise et acceptabilité sociale difficilement explicites et rarement partagés par l'ensemble des membres du collectif. Nous tentons ici de retracer le cheminement de son édification.

Après les premières séances du COMOP, qui avaient pour objectif de faire un état de l'art des connaissances scientifiques disponibles, le ministère a souhaité s'entourer d'un groupe d'experts scientifiques piloté par le Cemagref. Le MNHN, l'ONEMA ou le SETRA ont donc participé de manière ponctuelle pour des missions précises directement en lien avec leur domaine de compétence ${ }^{7}$, et $c^{\prime}$ est principalement le Cemagref qui a fait

6 Notes personnelles de R. Vimal, COMOP, réunion plénière du 21 janvier 2008, ministère de l'Écologie, du Développement et de l'Aménagement durables (MEDAD).

7 L'ONEMA a plus particulièrement travaillé sur la trame bleue, le SETRA sur le volet 3 du guide, portant spécifiquement sur les infrastructures, et le MNHN sur les listes d'espèces TVB déterminantes. 
figure de coordinateur scientifique au sein du dispositif. Ainsi, le suivi du travail effectué par le Cemagref donne à voir comment «le » scientifique peut directement être mobilisé dans ces dispositifs participatifs. Quelle mission lui accorde-t-on ? Quelle légitimité de participation et quelle liberté de décision ? Comment, en retour, il se positionne, adapte son discours et construit lui-même sa représentation de l'objet en question ? Il faut mentionner l'enjeu primordial relatif au domaine de compétence $\mathrm{du}$ référent scientifique. Le choix d'un ingénieur du Cemagref spécialiste en télédétection n'est pas anodin. D'une part, le Cemagref est un organisme public de recherche appliquée en sciences et technologies de l'environnement, dont certaines missions sont clairement en appui aux pouvoirs publics. D'autre part, le choix d'un télédétecteur témoigne des prédispositions du ministère à l'identification spatiale de la TVB. Si le choix d'un seul interlocuteur ${ }^{8}$ peut se justifier pour le ministère, on ne peut que constater le manque de complémentarité en termes de compétences scientifiques. La présence d'autres chercheurs, notamment en écologie, en sociologie, en droit, en géographie et en aménagement du territoire, aurait sans doute introduit un débat sur ce modèle et fait ressortir des enjeux non pris en considération - en tout cas de façon explicite -, tant sur le plan écologique (Boitani et al., 2007; Van der Windt et Swart, 2008) que sociologique (Mougenot et Melin, 2000 ; Fortier, 2009), géographique (Mathevet et al., 2010) ou juridique (Bonnin, 2008).

Si le Cemagref a été chargé de la rédaction du guide méthodologique en qualité d'organisme de recherche scientifique, il a effectué un travail de traduction (Callon, 1986) entre les mondes scientifiques et sociaux. La rédaction des trois volets s'est en effet construite selon un processus itératif au cours duquel le représentant du Cemagref a fait des propositions soumises au ministère et débattues ensuite au sein du COMOP. Pendant et après chaque réunion, les acteurs étaient invités à faire part de leurs commentaires et propositions de modifications. Les débats ont dès lors rapidement témoigné de la difficulté à discerner ce qui relève de la " science » ou du politique au sein d'un tel forum hybride. Lors d'un entretien, le représentant du Cemagref nous expliqua qu'il devait filtrer les différentes propositions, en traitant celles relevant de compétences scientifiques et en laissant au ministère le soin de l'arbitrage concernant des propositions d'ordre plus politique. C'est ainsi que, tout au long du processus décisionnel, les constituants scientifiques de la TVB - les connaissances de mode 1 (Nowotny et al., 2001), qui résultent déjà des compétences

\footnotetext{
8 Notons cependant que, pour accompagner la rédaction du guide, le représentant du Cemagref a recruté un ingénieur contractuel en écologie.
}

disponibles - sont traduites dans un langage permettant de rallier au projet de TVB des acteurs aux intérêts et positionnements stratégiques hétérogènes. Le maintien du soutien essentiel du ministère a demandé en particulier un travail de recadrage du projet, conforme aux exigences du fonctionnement du monde politique. Le rôle de coordinateur scientifique du Cemagref se révèle donc être celui d'un passeur entre les mondes scientifiques et politiques.

À la fin du mandat du COMOP, le ministère a organisé deux réunions où seuls des scientifiques académiques étaient conviés, afin de leur présenter le dispositif TVB proposé et ainsi légitimer scientifiquement le fruit de la démarche. Les représentants du monde scientifique alors mobilisés n'étant pas ceux présents dans le processus de traduction issu du COMOP, le bilan de ces réunions mentionne sans surprise les lacunes de connaissances et le fait que certains points ne font pas consensus au sein de la communauté scientifique. Des ajustements à la marge ont été effectués dans le guide méthodologique ${ }^{9}$. Cette forme de validation par des scientifiques non investis au départ dans le processus de traduction, et les ajustements qui ont suivi, montrent que le processus de traduction vers un projet politique n'a pas été totalement assumé par les opérateurs.

Finalement, la mobilisation de l'expertise scientifique, l'expertise formelle et mandatée, l'expertise académique en l'occurrence, s'est opérée sur deux modes disjoints qui semblent l'avoir affaiblie. D'une part, au sein du groupe d'experts du COMOP, elle a dû entrer dans un processus de traduction qui, par nature, «établit une équivalence toujours contestable entre des problèmes formulés par plusieurs acteurs dans des répertoires différents" (Callon, 1988). À la charnière entre les différents acteurs, le Cemagref a de fait endossé le rôle de principal opérateur de traduction entre ces acteurs et le ministère, son rôle de représentant purement scientifique se voyant alors moins reconnu. D'autre part, des interventions ponctuelles de scientifiques ont été mobilisées de façon parallèle, rarement en présence de l'ensemble des acteurs. Dans ce cas, le mécanisme qui opère le passage de connaissances de mode 1, complexes et incertaines, à des connaissances situées, pragmatiques et politisées n'est pas ou peu partagé.

\footnotetext{
9 À titre d'exemple, le guide mentionne en avertissement (Allag-Dhuisme et al., 2010b, p. 24) : « Pour la sélection des espèces dites déterminantes TVB pour les plantes vasculaires : l'adaptation de la méthodologie a étéjugée nécessaire lors du séminaire d'experts scientifiques du 4 mars 2010, animé par le MNHN. Une première note de problématique a été établie par la fédération des conservatoires botaniques nationaux et communiquée aux CSRPN avec les pré-listes de faune transmises en avril 2010. Les travaux restent à poursuivre sur cette méthodologie, en lien avec les réflexions sur l'entomofaune et les habitats déterminants TVB.»
} 


\section{De l'asymétrie de pouvoir à l'expertise environnementale}

Si, lors de la première réunion du COMOP, le sénateur président de séance déclarait: « Je souhaite que nous nous donnions le temps et les moyens d'élaborer une vision partagée de ce que devrait être la $\mathrm{TVB}^{10}$ », deux ans après, nous constatons que cet objectif collectif est indissociable de la recherche permanente, de la part des différents acteurs en présence, d'une reconnaissance de leur légitimité respective. Dès lors, il s'agit de comprendre comment la TVB et les outils proposés pour sa mise en œuvre peuvent être avant tout un construit social qui, d'une part, résulte des interactions plus ou moins conflictuelles entre les participants et, d'autre part, produit et structure dans le même temps leurs idées et représentations. Et si le sénateur ajoutait: « [...] au sein de notre COMOP, nous sommes tous à égalité », on ne peut que constater l'hétérogénéité du groupe en termes de participation et d'investissement. Évidemment, tous les acteurs représentés dans ce forum n'ont pas les mêmes intérêts à y défendre ni les mêmes ressources à mobiliser. Lors de l'une des premières réunions, le directeur de la FCEN comparait la position de sa structure, où lui seul devait prendre le temps de préparer les réunions, avec celle de FNE, où deux personnes travaillaient à temps plein sur le sujet. Dans ce contexte, la question de la gestion du temps a pris toute son importance et, dès le début, l'ensemble des acteurs déplorait les contraintes de temps qui obligeaient le groupe à respecter un planning imposé non pas par ses propres missions, mais par les échéances législatives. Procédure chronophage et parisienne, le COMOP opérait alors une première sélection des acteurs.

Très rapidement, la dynamique collective du COMOP s'est polarisée autour de FNE et des organisations professionnelles agricoles (OPA), à savoir l'APCA et la FNSEA. La participation à des groupes de travail en parallèle aux réunions du COMOP a joué un rôle important dans la structuration du réseau d'acteurs autour de diverses alliances et a été, pour certains d'entre eux, un moyen d'exister et d'influencer le dispositif résultant. La FPNR et la FCEN ont, par exemple, réalisé conjointement une étude sur les mesures contractuelles potentiellement mobilisables dans le cadre de la TVB. De leur côté, FNE, l'APCA, la FNSEA et FPF ont mené une réflexion commune sur les enjeux socioéconomiques liés à la TVB. Au cours du temps, les groupes de travail et réunions annexes ont par ailleurs pris de plus en plus de place aux dépens des réunions plénières, multipliant les lieux de discussion, de confrontation ou de partage des points de vue. C'est sur le ton de l'ironie que, lors d'une discussion visant

\footnotetext{
10 Notes personnelles de R. Vimal, COMOP, réunion plénière du 21 janvier 2008, MEDAD.
}

à établir la composition de nouveaux groupes de travail, la représentante de l'APCA lançait à son homologue de FNE : «Tout sera décidé dans les groupes de travail, donc il faudra que tu sois dans tous les groupes ${ }^{11}$. »

Bien sûr, on ne peut considérer le COMOP de manière isolée. En décidant de mettre en œuvre la TVB, le gouvernement répond à un engagement pris au sein du Conseil de l'Europe de participer à la création d'un Réseau écologique paneuropéen (Council of Europe $\mathrm{et}$ al., 1996). La définition de la TVB ainsi validée au sein du COMOP est d'ailleurs tout à fait conforme à celle communément admise dans d'autres pays d'Europe. Bénéficiant en début de mandat de tout un retour d'expériences assuré par divers porteurs de projet à des échelles variées $^{12}$, le COMOP n'a été, semble-t-il, ni un lieu de questionnement de ce modèle de représentation ni un lieu d'exploration d'autres approches. Au contraire, la représentation de la TVB telle que proposée a été largement mise en avant par certains acteurs, qui ont dès lors pu mobiliser des ressources devenues stratégiques dans le cadre du Grenelle de l'environnement. Il s'agit de la FPNRF, qui au début du COMOP a multiplié les interventions pour faire valoir son expertise sur les questions de TVB. Depuis plusieurs années, de nombreux parcs naturels régionaux travaillaient en effet sur la thématique (Girault, 2005) et la FPNRF animait un " groupe corridor », rebaptisé pour l'occasion « groupe TVB». Mais il s'agit aussi de FNE, qui avait déjà rédigé et diffusé un document de réflexion sur les réseaux écologiques (Pavard et Paquin, 2006) allant dans ce sens et qui, au travers du COMOP et en amont, s'est « imposée » en " transcodeur » (Lascoumes, 1994) de la TVB. Les tables rondes du Grenelle ont en effet représenté une véritable fenêtre politique (Kingdom, 1984 ; Keeler, 1993) pour la fédération, qui, depuis plusieurs années déjà, militait pour l'établissement d'un réseau écologique national.

Dans ce contexte, nous faisons l'hypothèse que l'effacement de l'expert scientifique reconnu comme tel au sein $\mathrm{du}$ dispositif a pu permettre à l'expertise associative incarnée par FNE d'occuper l'espace vacant et d'affirmer sa légitimité. Celle-ci repose moins sur un niveau de

\footnotetext{
11 Notes personnelles de R. Vimal, COMOP, réunion plénière 16 décembre 2008, ministère de l'Écologie, de l'Énergie, du Développement durable et de l'Aménagement du territoire (MEEDDAT).

12 Liste non exhaustive : mise en œuvre du réseau Isérois (conseil général de l'Isère); TVB en Alsace (conseil régional d'Alsace) ; Réseau routier et continuité écologique (Autoroutes du Sud de la France [ASF]) ; Trame verte et carrière (Union nationale des industries de carrières et matériaux de construction [UNICEM]) ; Réseau électrique et TVB (Réseau de transport d'électricité [RTE]) ; Expérience, méthodologie et mise en œuvre (ECONAT, bureau d'étude en écologie appliquée) ; Apport des expériences européennes pour la mise en place régionale (conseil régional de Lorraine).
} 
connaissance validé que sur l'appartenance à une communauté (Lavignotte-Guérin, 1999), en l'occurrence ici celle des experts de la nature. En effet, c'est en tant que promoteur de la TVB bien en amont du Grenelle que la fédération a progressivement bénéficié du rôle d'expert. Elle a participé activement à tous les groupes de travail, fait des propositions d'ordre du jour pour les séances plénières, rédigé de nombreuses notes à l'attention du Cemagref, etc. Des réunions spécifiques ont été organisées en marge du COMOP entre le ministère, FNE et le groupe d'experts. Notons ici que ce rapport privilégié entre FNE et le ministère n'est pas propre à ce dispositif. Certains auteurs (Lascoumes, 1994 ; Charvolin, 2003) ont montré que les associations naturalistes ont toujours été le bras armé d'un ministère faible en moyens et toujours en quête de légitimité. Cet échange stabilisé de longue date entre FNE et le ministère est donc ici un élément central de l'approche, puisqu'il suppose qu'au travers du COMOP, les deux partenaires ont tenté de renforcer leur capacité d'action en assurant leur objectif commun que représente le succès du Grenelle. Dans un champ politique où les rapports de force sont traditionnellement très favorables aux acteurs économiques, le Grenelle del'environnement constitue une fenêtre d'opportunité sans précédent pour les acteurs environnementaux. Le passage dans le champ politique suppose alors un recadrage correspondant à ses règles propres (Kingdom, 1984), rendant indispensables certaines simplifications, comme l'illustre le travail de FNE. Ainsi, lors d'une discussion en marge d'une réunion du COMOP, le représentant de FNE, au sujet de l'identification cartographique de grandes continuités naturelles à l'échelle de la France, nous assurait comprendre que la réalité écologique était certainement plus complexe, mais que cela restait un moyen de limiter les nouvelles infrastructures linéaires de transport. Le rôle joué par la fédération peut aussi être illustré par un exemple tout à fait révélateur et qui a pris une certaine place dans les débats : une fois la représentation de la TVB admise, FNE a proposé au ministère $\mathrm{d}^{\prime}$ utiliser des listes d'espèces régionales comme un critère de cohérence nationale pour mettre en œuvre la TVB. Bien quel'approche ait été perçue comme complexe et peu pratique par les parties prenantes, le ministère, désireux de ne pas compromettre la démarche par un retrait de FNE, a mandaté le MNHN pour définir une méthode permettant d'identifier ces listes d'espèces, ordinaires ou remarquables, vis-à-vis desquelles la région a une responsabilité élevée et dont le maintien nécessiterait de préserver les continuités écologiques. Forts de leur expérience du territoire, les acteurs directs de la conservation, notamment la FPNRF, dont le positionnement s'est largement construit sur la base des réflexions en cours au niveau du "groupe TVB», ont manifesté leur crainte d'une démarche trop technique qui pourrait compromettre l'adhésion des acteurs. La consultation nationale en ligne portant sur les guides méthodologiques à mimandat a d'ailleurs permis aux experts et praticiens de terrain de manifester des positions proches de celles de la FPNRF. FNE s'est donc retrouvée isolée, et certains représentants des autres structures ont jugé sa posture trop lobbyiste et trop peu consensuelle. Finalement, les listes d'espèces TVB déterminantes ont été maintenues dans le dispositif, mais leur importance semble avoir été largement minimisée : elles sont le résultat d'un compromis entre l'efficacité et l'assurance juridique voulue par FNE, l'enjeu de la complexité technique et de l'acceptation sociale défendue par bon nombre d'acteurs et le débat scientifique de fond alimenté par les spécialistes et qui oppose entrée " espèces » et entrée « espaces ».

\section{Du cognitif aux instruments d'action publique}

D'après notre analyse, il apparaît que les acteurs non avertis du COMOP n'ont pas vraiment pu agir sur la définition de la TVB non pas par inintérêt, mais plutôt parce qu'ils sont intervenus à un moment où celle-ci constituait déjà une "boîte noire » : la complexité technique menait alors à l'exclusion des profanes de toute discussion. Paradoxalement, l'absence de controverse autour du modèle proposé s'explique aussi par la flexibilité et le flou qui le caractérisent. En effet, si la représentation schématique semble avoir des vertus pédagogiques, il s'avère difficile de se rendre compte de la forme que prendra la TVB sur un territoire donné et donc des contraintes et avantages qu'elle engendrera. C'est la force des images, qui agissent comme des slogans mobilisateurs, d'autant mieux qu'elles évacuent les points de conflit potentiels (Muller, 1984). Dans le cas présent, ce travail de traduction n'a pas totalement fonctionné. On constate des « débordements » de certains participants (Callon, 1986) qui expriment des frustrations et des questionnements, comme l'illustre cette intervention du représentant de l'APCA qui, lors de l'une des dernières réunions, interroge la stabilité du consensus soi-disant assurée par le modèle : « Il y a deux Trames, celle qu'on a définie et puis vous êtes en train de parler de tout un tas d'autres continuités qu'on peut prendre en compte dans les SCOT ${ }^{13} »$.

Devant cet écueil, il semble que les acteurs invités à participer aient considéré progressivement qu'au-delà de la définition donnée de la TVB, largement laissée à la charge des structures les plus "spécialistes », ce qui faisait sens reposait plutôt sur la sémantique et le discours relatif à son implantation dans le tissu territorial local, ainsi que sur les outils de sa mise en œuvre, les instruments de la

\footnotetext{
13 SCOT : schémas de cohérence territoriale. Notes personnelles de R. Vimal, COMOP, 17 décembre 2009, MNHN.
} 
nouvelle politique publique en question. Le texte de loi, mais surtout le guide méthodologique, rendent bien compte des jeux d'acteurs qui ont animé le dispositif au cours de ces deux années. Les OPA, représentées par deux juristes appartenant à ces structures, ont rapidement fait alliance pour assurer un contrepoids face à l'ambition des environnementalistes et veiller à minimiser l'impact de la politique TVB. Elles ont notamment été très vigilantes quant à la traduction juridique des éléments des trois volets du guide et ont insisté sur des aspects sémantiques, essayant à tout prix d'éviter les rapprochements avec des démarches récoltant une opinion négative auprès du monde agricole. Il s'agit pour elles de ne pas apparaître comme un facteur de résistance, voire d'échec, au projet tout en maintenant leur légitimité en tant que porteparole des agriculteurs. Ainsi, dès la phase des tables rondes du Grenelle, le nom de " réseau écologique » a été remplacé par « trame verte et bleue » car jugé trop proche du « réseau Natura 2000 », dont la mise en place avait été très contestée par les acteurs de l'espace rural. Pour désigner les zones à haute valeur écologique, l'expression « réservoir de biodiversité » a été préférée à celle de " cœur de nature ", qui ne se distinguait pas suffisamment des «cœurs » de parcs nationaux. Au-delà des oppositions, grâce au dispositif COMOP, certains éléments sontl'occasion pour le collectif d'affirmer une unité à travers une vision commune des objectifs de la TVB. À titre d'exemple, un paragraphe intitulé « La Trame verte et bleue créatrice d'emploi » a été largement soutenu par l'ensemble des acteurs et mentionne que « la Trame verte et bleue doit être une politique d'aménagement du territoire en faveur des habitants. Prenant en compte les atouts et les fragilités des territoires, elle doit permettre de préserver et développer au mieux leur équilibre et leurs potentialités économiques » (Allag-Dhuisme et al., 2010a, p. 32).

$C^{\prime}$ est donc sous cet angle que chacun des acteurs s'est positionné dans le dispositif pour que les moyens qui serviront à mettre en œuvre la TVB soient les plus efficaces pour certains, les moins contraignants pour d'autres. De ces positions divergentes sont nées les plus vives controverses, comme le laisse entendre cette intervention de FPF : «Beaucoup de zonages apparaissent. Je rappelle $\mathrm{qu}^{\prime}$ on ne souhaite pas, au niveau forestier, voir s'étendre le nombre de zonages. Enfin, nous sommes très attachés à la contractualisation, mais on veut savoir que prendre en compte dans cette contractualisation, les limites de cette contractualisation et enfin qui va payer, sachant que me dire que ce sont les collectivités n'est pas une réponse pertinente ${ }^{14}$. » Ainsi, comme l'ont montré Lascoumes et LeGalès (2005), les choix d'instruments d'action publique ne relèvent pas de « simples choix techniques », mais sont

\footnotetext{
14 Notes personnelles de R. Vimal, COMOP, $1^{\mathrm{er}}$ avril 2010, Association immobilière de l'École militaire (ASIEM).
}

avant tout le résultat de logiques politiques. Si la contractualisation est un moyen de faire bénéficier d'un revenu leur réseau, agriculteurs comme forestiers ont réagi vivement à toutes les injonctions et recommandations de gestion qui risquaient de porter atteinte à leur souveraineté. La question de l'opposabilité, et donc du zonage, est à l'origine d'une opposition majeure entre les deux principaux protagonistes : FNE militait pour que la TVB soit opposable dans les documents d'urbanisme, alors que les OPA refusaient toute forme d'approche réglementaire qui inclurait les terres agricoles. Incapable de trouver un compromis sur ces questions, le COMOP a renvoyé la controverse à un arbitrage par les élus des assemblées, terrain de discussion plus opaque et moins favorable structurellement aux acteurs environnementaux. De fait, le choix a finalement été fait d'une opposabilité faible de la TVB.

\section{Conclusion}

Après la nature, l'environnement puis la biodiversité, le concept de réseau écologique est comme l'aboutissement du processus d'écologisation des politiques publiques. La TVB traverse, requalifie le territoire et souligne à la fois l'enjeu de la réflexion sur les représentations de son fonctionnement écologique et l'enjeu d'un compromis social pour relier les composantes garantes de ce fonctionnement. On observe ainsi un double débat au sein du COMOP : celui relatif à la conception de la TVB et celui relatif à son application sur le terrain. Ces deux modes de penser et d'agir se mélangent dans le processus d'élaboration de la politique de TVB, dont l'analyse critique pose la question de la relation entre science et action publique : Comment l'intervention de l'État se fonde-t-elle sur l'expertise? Nous avons vu que le modèle support de représentation de la TVB repose sur de nombreuses simplifications et autant d'incertitudes, inhérentes à tout processus de traduction. Ces dernières sont-elles réellement reconnues et prises en charge par le dispositif participatif du Grenelle de l'environnement?

Le produit du COMOP bénéficie d'une légitimité de type procédural liée à son élaboration collective. Cependant, si l'organisation délibérative enrôle des acteurs issus de différents réseaux sociotechniques, la création de nouvelles règles ne s'est pas accompagnée d'une mise en débat du principe même de TVB. Le COMOP ne peut donc être considéré comme un forum associant connaissances de mode 1, issues plutôt d'une activité scientifique en tant que telle, et connaissances de mode 2 , auxquelles chaque acteur présent est en capacité de participer.

Si la socialisation des énoncés et la traduction des argumentaires de chacun ont demandé des ajustements dans la définition de ce qu'est chaque acteur, le COMOP a été surtout un lieu de négociation au sein duquel les 
groupes d'intérêt n'ont accordé que peu d'importance au cadre cognitif de la politique publique et ont focalisé plutôt sur son volet normatif et sur les instruments de sa mise en œuvre. Ainsi, le processus de définition de ce qu'est la TVB a semblé échapper au collectif pour être pris en charge par les acteurs les plus spécialistes. Le dispositif d'action publique proposé est profondément ancré dans une démarche technique qui nous interroge, d'une part, sur l'acceptabilité sociale qui en résultera une fois confronté aux réalités du territoire et, d'autre part, sur l'efficacité et la pertinence écologique d'une telle démarche.

Cette étude discute le rôle, les objectifs et l'efficacité attendue de ces dispositifs participatifs temporaires. Nous avons pu constater en effet que le COMOP n'était pas une instance isolée et que la définition de la TVB et du cadre $\mathrm{d}$ 'action publique qui en résulte est largement dépendante d'interactions, de jeux d'acteurs, de contraintes historiques ou encore d'un cadre législatif supranational qui dépassent largement l'arène participative et questionnent son autonomie. À l'avenir, pour donner plus de sens au collectif, pour qu'il fasse davantage " cause commune » (Stengers, 2002), il semble que la place accordée aux connaissances de mode 1 , et donc à l'expertise académique, puisse jouer un rôle déterminant. Nous faisons ici l'hypothèse que c'est le manque d'implication des scientifiques ou d'experts reconnus en tant que tels, l'absence de complémentarité des compétences ou des conditions favorables à leur mobilisation qui n'ont pas permis au collectif de partager l'incertitude et la complexité des connaissances relatives à la notion de réseau écologique pour créer avant tout les conditions d'une vision commune (Charvolin et al., 2011) et rendre viable une certaine rationalité communicationnelle (Habermas, 1987).

Malgré tout, le dispositif existe. Les acteurs représentant l'environnement ont ainsi disposé d'une fenêtre d'opportunité pour faire avancer les choses. Évidemment, en entrant dans le champ politique, ce dernier impose un formatage relationnel et des rapports de force qui limitent les possibilités d'action. Le processus apparaît alors comme le début d'une construction progressive d'une représentation partagée du problème de la gestion intégrée des territoires de la biodiversité, des solutions et de leurs conséquences. Il produit un nouveau cadre normatif d'action combinant des éléments de puissance publique et des éléments d'expertise qui devraient donner lieu à des progrès et rebonds au cours des années à venir.

\section{Remerciements}

Nous remercions l'équipe du ministère en charge de l'Écologie pour son accueil ainsi que tous les membres du COMOP TVB. Merci également à Jennifer Amsallem et Michel Deshayes (IRSTEA) pour nos nombreux échanges, ainsi qu'à Thierry Mougey (FPNR) pour ses commentaires avisés sur le travail présenté ici.

\section{Références}

Allag-Dhuisme, F., Amsallem, J., Barthod, C., Deshayes, M., Graffin, V., Lefeuvre, C., Salles, É. (Eds), 2010a. Choix stratégiques de nature à contribuer à la préservation et à la remise en bon état des continuités écologiques : premier document en appui à la mise en cuvre de la Trame verte et bleue en France. Proposition issue du comité opérationnel « Trame verte et bleue », MEEDDM, Paris.

Allag-Dhuisme, F., Amsallem, J., Barthod, C., Deshayes, M., Graffin, V., Lefeuvre, C., Salles, É. (Eds), 2010b. Guide méthodologique identifiant les enjeux nationaux et transfrontaliers relatifs à la préservation et à la remise en bon état des continuités écologiques et comportant un volet relatif à l'élaboration des schémas régionaux de cohérence écologique : deuxième document en appui à la mise en cuvre de la Trame verte et bleue en France. Proposition issue du comité opérationnel «Trame verte et bleue », MEEDDM, Paris.

Allag-Dhuisme, F., Barthod, C., Bielsa, S., Brouard-Masson, J., Graffin, V., Vanpeene, S. (Eds), 2010c. Prise en compte des orientations nationales pour la préservation et la remise en bon état des continuités écologiques pour les grandes infrastructures linéaires de l'État et de ses établissements publics: troisième document en appui à la mise en cuvre de la Trame verte et bleue en France. Proposition issue du comité opérationnel « Trame verte et bleue », MEEDDM, Paris.

Barré, R., 2004. La Science est morte, vive la Science! Le nouveau contrat entre la science et la société postmoderne : l'avènement de la recherche de mode 2 , Natures Sciences Sociétés, 12, 1, 52-55.

Boitani, L., Falcucci, A., Maiorano, L., Rondinini, C., 2007. Ecological networks as conceptual frameworks or operational tools in conservation, Conservation Biology, 21, 6, 1414-1422.

Bonnin, M., 2008. Les Corridors écologiques : vers un troisième temps du droit de la conservation de la nature?, Paris, L'Harmattan.

Callon, M., 1986. Éléments pour une sociologie de la traduction. La domestication des coquilles Saint-Jacques dans la baie de Saint-Brieuc, L'Année sociologique, 36, 169-208.

Callon, M., 1988. La Science et ses réseaux : genèse et circulation des faits scientifiques, Paris, La Découverte.

Callon, M., Latour, B., 1991. La Science telle qu'elle se fait, Paris, La Découverte.

Callon, M., Lascoumes, P., Barthe, Y., 2001. Agir dans un monde incertain : essai sur la démocratie technique, Paris, Le Seuil.

Charvolin, F., 2003. L'Invention de l'environnement en France: chronique anthropologique d'une institutionnalisation, Paris, La Découverte.

Charvolin, F., Mathevet, R., Vimal, R., 2011. La Trame verte et bleue et son public, Quaderni, 76, 67-78.

ComMod, 2009. La posture d'accompagnement des processus de prise de décision: les références et les questions transdisciplinaires, in Hervé, D., Laloë, F. (Eds), Modélisation de l'environnement: entre natures et sociétés, Versailles, Quae / NSS-Dialogues, 71-89. 
Council of Europe, United Nations Environment Programme, European Information Centre for Nature Conservation, 1996. The Pan-European Biological and Landscape Diversity Strategy: A Vision for Europe's Natural Heritage, Strasbourg (France), Council of Europe / Geneva (Switzerland), UNEP / Tiburg (The Netherlands), ECNC.

Fortier, A., 2009. La conservation de la biodiversité. Vers la constitution de nouveaux territoires ?, Études rurales, 183, 129-142.

Friedberg, E., 1993. Le Pouvoir et la règle : dynamiques de l'action organisée, Paris, Le Seuil.

Funtowicz, S.O., Ravetz, J.R., 1993. Science for the post-normal age, Futures, 25, 739-755.

Girault, V., 2005. Mise en cuvre des corridors écologiques et/ou biologiques sur le territoire des parcs naturels régionaux: définition d'une méthodologie commune et recueil d'expériences. Rapport de stage "éco-conseiller », Fédération des parcs naturels régionaux de France, pôle Développement durable, [Paris].

Habermas, J., 1973. La Technique et la science comme « idéologie », Paris, Gallimard.

Habermas, J., 1987. Théorie de l'agir communicationnel, Paris, Fayard.

Jobert, B., 1994. Le Tournant néolibéral en Europe, Paris, L'Harmattan.

Keeler, J., 1993. Opening the window for reform, Comparative Political Studies, 25, 4, 433-448.

Kingdom, J., 1984. Agendas, Alternatives and Public Policies, Boston (MAS), Little Brown.

Lascoumes, P., 1994. L'Éco-pouvoir : environnements et politiques, Paris, La Découverte.

Lascoumes, P., 2011. Des acteurs aux prises avec le «Grenelle Environnement ». Ni innovation politique, ni simulation démocratique, une approche pragmatique des travaux du Groupe V, Participations, 1, 277-310 (DOI : 10.3917/ parti.001.0277).

Lascoumes, P., Le Galès, P., 2005. Gouverner par les instruments, Paris, Presses de Sciences Po.

Lavignotte-Guérin, É., 1999. Expertise et politiques européennes de développement local, Paris, L'Harmattan.
Mathevet, R., Thompson, J., Delanoë, O., Cheylan, M., GilFourrier, C., Bonnin, M., 2010. La solidarité écologique : un nouveau concept pour la gestion intégrée des parcs nationaux et des territoires, Natures Sciences Sociétés, 18, 424-433.

MEDAD, 2007. Le Grenelle Environnement. Préserver la biodiversité et les ressources naturelles : synthèse et rapport du groupe 2 (http:/ / www.legrenelle-environnement.fr/IMG/ G2_Synthese_Rapport.pdf).

Mougenot, C., Melin, É., 2000. Entre science et action: le concept de réseau écologique, Natures Sciences Sociétés, 8, 3, 20-30.

Muller, P., 1984. Le Technocrate et le paysan, Paris, Éditions ouvrières.

Muller, P., 1985. Les Politiques publiques, Paris, PUF.

Muller, P., 1995. Les politiques publiques comme construction d'un rapport au monde, in Faure, A., Pollet, G., Warin, P., La Construction de sens dans les politiques publiques : débats autour de la notion de référentiel, Paris, L'Harmattan, 153-179.

Nowotny, H., Scott, P,. Gibbons, M., 2001. Re-Thinking Science: Knowledge and the Public in an Age of Uncertainty, Cambridge (UK), Polity Press.

Pavard, I., Paquin, M., 2006. Contribution à la constitution du Réseau écologique national: réflexions sur la constitution d'un réseau écologique en France, Paris, France Nature Environnement.

Roqueplo, P., 1997. Entre savoir et décision, l'expertise scientifique, Paris, INRA.

Stengers, I., 2002. Sciences et pouvoirs : la démocratie face à la technoscience, Paris, La Découverte.

Touraine, A., 1980. La Prophétie anti-nucléaire, Paris, Le Seuil.

Van der Windt, H.J., Swart, J.A.A., 2008. Ecological corridors, connecting science and politics: The case of the Green River in the Netherlands, Journal of Applied Ecology, 45, 124-132.

Vimal, R., Mathevet, R., Thompson, J.D., 2012. The changing landscape of ecological networks, Journal for Nature Conservation, 20, 49-55.

Weber, M., 1959. Le Savant et le politique, Paris, Plon.

Reçu le 21 octobre 2010. Accepté le 6 octobre 2011. 